\title{
Avaliação da microfiltração para remoção do lodo gerado no processo oxidativo avançado empregando o reagente de Fenton no tratamento de lixiviado de aterro sanitário
}

\section{Evaluation of microfiltration for removal of sludge generated in advanced oxidation process by Fenton reagent in treatment of landfill leachate}

\author{
Wagner Guadagnin Moravia \\ Engenheiro Civil, Mestre e Doutor em Saneamento, Meio Ambiente e Recursos Hídricos pela Universidade Federal de Minas Gerais (UFMG)
}

\author{
Liséte Celina Lange \\ Química, Doutora em Tecnologia Ambiental pela Universidade de Londres, Inglaterra. Professora Associada do Departamento de Engenharia Sanitária e \\ Ambiental da UFMG

\section{Míriam Cristina Santos Amaral} \\ Engenheira Química, Doutora em Saneamento, Meio Ambiente e Recursos Hídricos pela UFMG. Professora Adjunta do Departamento de Engenharia \\ Sanitária e Ambiental da UFMG
}

\section{Resumo}

Uma alternativa atrativa para o tratamento de lixiviado de aterro sanitário são os processos oxidativos avançados empregando o reagente de Fenton (POA/ Fenton). No entanto, a aplicação do POA/Fenton é limitada pela geração de lodo no processo. Esse lodo possui elevada concentração de ferro, o que torna necessária sua separação do efluente tratado. O objetivo desse trabalho foi avaliar a microfiltração para a remoção de lodo gerado no POA/Fenton. A pesquisa foi desenvolvida em unidade de bancada. O POA/Fenton apresentou elevada eficiência na remoção de matéria orgânica (75\%) e cor real (95\%). O processo de microfiltração foi eficiente na separação do lodo gerado, o qual apresentou baixa sedimentabilidade, dificultando sua separação por sedimentação, além de ter contribuído para a remoção de outros poluentes.

Palavras-chaves: microfiltração; lodo de processo oxidativo avançado; reagente de Fenton; lixiviado de aterro sanitário.

\section{Abstract}

Advanced oxidation processes using Fenton's reagent (AOP/Fenton) are an attractive alternative for landfill leachate treatment. However, the implementation of the AOP/Fenton is limited by the generation of sludge in the process. The sludge has a high iron concentration, which needs its separation from the treated effluent. The aim of this study was to evaluate the microfiltration for sludge removal from an AOP/Fenton. The work was conducted in bench scale. The advanced oxidation process showed high efficiency in removing organic matter (75\%) and true color (95\%). The microfiltration was effective in separating the sludge, which showed low settling hindering their separation by sedimentation, and has contributed to the removal of other pollutants.

Keywords: microfiltration; sludge of advanced oxidative process; Fenton's reagent; landfill leachate. 


\section{Introdução}

Os processos mais usuais para o tratamento de lixiviados de aterro sanitário são os biológicos, que têm se mostrado insuficientes para o atendimento aos padrões de lançamento estabelecidos pela legislação, principalmente no caso de lixiviados de aterro sanitário estabilizados. Uma alternativa para esse tipo de lixiviado são os tratamentos baseados em processos químicos capazes de promover a degradação ou até mesmo a mineralização da matéria poluente refratária, podendo haver a combinação entre diferentes técnicas ou serem conjugados com processos biológicos (KURNIAWAN et al., 2006).

Os processos oxidativos avançados ( $\mathrm{POA}$ ) envolvem a geração de radicais hidroxilas $(\cdot \mathrm{OH})$, altamente reativos, que têm a capacidade de destruição total ou parcial de muitos poluentes orgânicos. Os radicais livres $(\cdot \mathrm{OH})$ formados atacam, de forma não seletiva, os compostos orgânicos levando à sua oxidação completa produzindo $\mathrm{CO}_{2}$ e $\mathrm{H}_{2} \mathrm{O}$ ou, quando resultam em uma oxidação parcial, geralmente ocorre um aumento da biodegradabilidade dos poluentes. A transformação dos poluentes pela oxidação pode favorecer o emprego de técnicas físico-químicas ou biológicas de tratamento complementar (BULL e ZEFF, 1991).

$\mathrm{O}$ POA pelo reagente de Fenton ( $\mathrm{POA} /$ Fenton) vem sendo extensivamente estudado nos últimos anos e tem sido considerado a técnica de melhor custo-benefício entre os POA (ENGLEHARDT et al., 2006). No POA pelo reagente de Fenton (POA/Fenton), o peróxido de hidrogênio $\left(\mathrm{H}_{2} \mathrm{O}_{2}\right)$ é empregado para geração de radicais hidroxilas, entretanto, é necessária a adição de ativadores, como sais de ferro, para produzir a alta taxa de radicais requerida (LI et al., 2010). A reação ocorre em um reator simples, sem a necessidade de equipamentos complexos e condições extremas de temperatura e pressão. Outra vantagem do reagente de Fenton é sua menor complexidade operacional em relação a outras técnicas de $\mathrm{POA}$, como os processos fundamentados em ozônio e radiação ultravioleta, que necessitam de gerador de $\mathrm{O}_{3}$ e fonte ultravioleta, respectivamente (CHAMARRO et al., 2001). As Reações 1, 2, 3 e 4 mostram de forma simplificada o mecanismo de reação da decomposição catalítica do $\mathrm{H}_{2} \mathrm{O}_{2}$ por sais de ferro, em meio ácido.

$$
\begin{aligned}
& \mathrm{Fe}^{2+}+\mathrm{H}_{2} \mathrm{O}_{2} \rightarrow \mathrm{Fe}^{3+}+\mathrm{OH}^{-}+\mathrm{HO}^{\bullet} \quad \text { (início da cadeia de reação) } \\
& \mathrm{RH}+\mathrm{HO}^{\bullet} \rightarrow \mathrm{R}^{\bullet}+\mathrm{H}_{2} \mathrm{O} \\
& \mathrm{R}^{\bullet}+\mathrm{Fe}^{3+} \rightarrow \mathrm{R}^{+}+\mathrm{Fe}^{2+} \\
& \mathrm{Fe}^{2+}+\mathrm{HO}^{\cdot} \rightarrow \mathrm{Fe}^{3+}+\mathrm{OH}^{-} \quad \text { (final da reação) }
\end{aligned}
$$

Onde:

$\mathrm{Fe}^{2+}$ e $\mathrm{Fe}^{3+}$ representam as espécies hidratadas, $\mathrm{Fe}\left(\mathrm{H}_{2} \mathrm{O}\right)_{6}{ }^{2+} \mathrm{e}$ $\mathrm{Fe}\left(\mathrm{H}_{2} \mathrm{O}\right)_{6}{ }^{3+}$, respectivamente. A Reação 1 é conhecida como a reação de Fenton, embora muitas outras reações ocorram no sistema.

Entretanto, a aplicação do POA/Fenton é limitada pela geração de lodo no processo, o qual possui elevada concentração de ferro, tornando necessária sua separação do efluente tratado. Convencionalmente, emprega-se uma etapa de neutralização/precipitação para a separação do lodo gerado, na qual se exigem tempos elevados, superior a 2 horas, em função da baixa sedimentabilidade desse lodo, incrementando significativamente o tempo do tratamento (MORAVIA, 2010).

A conjugação do POA/Fenton com a microfiltração (MF) apresenta diversas vantagens. A etapa de decantação utilizada na separação do lodo pode ser eliminada com o uso da MF, diminuindo drasticamente o tempo de tratamento. Essa associação também permite uma maior eficiência de remoção de poluentes, por oferecer tratamento complementar visando ao enquadramento do efluente segundo os padrões da legislação vigente, além de economia nos requisitos de área para a implementação da planta de tratamento, característica esta evidente dos processos de separação por membranas quando comparadas com técnicas convencionais de tratamento (MORAVIA, 2010; HABERT et al., 2006).

A MF pode ser entendida como processo que envolve a utilização de membranas sintéticas, com diâmetro de corte de $10^{-1}$ a $10 \mu \mathrm{m}$, para a separação de componentes presentes (BAKER, 2004). Habert et al. (2006) definem membrana como uma barreira que separa duas fases e que restringe, sob a ação de uma força motriz, total ou parcialmente, o transporte de uma ou várias espécies químicas presentes nessas fases. As moléculas de tamanho inferior ao do poro da membrana passam através dela, enquanto as de tamanho superior ficam retidas pelo efeito de retenção ou por forças repulsivas atuantes na superfície da membrana. A alimentação separa-se, assim, em duas correntes: o fluido que atravessa a membrana, chamado de filtrado ou permeado, e o que permanece ao lado da alimentação que contém os solutos ou sólidos suspensos que foram rechaçados pela membrana, denominado de concentrado ou retido (ORDÓNEZ, 2005). Tais operações baseiam-se na permeabilidade seletiva de um ou mais componentes através da membrana.

A incrustação das membranas, em algumas vezes, tem sido um fator limitante para a aplicação dos processos de separação por membranas. Esse fenômeno condiciona o declínio do fluxo permeado com o tempo, fazendo com que o fluxo se torne independente da pressão através da membrana. A seleção adequada das características da alimentação, das condições hidrodinâmicas do sistema e das técnicas de limpeza, pode ser usada para minimizar a incrustação.

Ainda em relação às vantagens da conjugação de POA/Fenton com MF, em sinergia, o POA/Fenton também atua na minimização da incrustação da membrana de MF, uma vez que, durante a reação de oxidação, ocorre coagulação das partículas coloidais, o que pode reduzir a penetração dessas partículas dentro dos poros da membrana, aumentando-se assim o fluxo sustentável por meio de modificações das características do material depositado (CHIU E JAMES, 2006).

Dessa forma, o objetivo deste trabalho foi avaliar a MF na remoção de lodo gerado no processo oxidativo avançado pelo reagente de Fenton no tratamento de lixiviado do aterro sanitário da cidade de Belo Horizonte (MG). 


\section{Metodologia}

\section{Amostragem}

O lixiviado utilizado para a realização dos experimentos proveio do Aterro Sanitário da Central de Tratamento de Resíduos Sólidos (CTRS) da cidade de Belo Horizonte. O aterro ocupa uma área de 133 hectares, possui células de aterramento com diferentes idades e características e encontra-se com sua atividade de recebimento de resíduos sólidos urbanos encerrada. O lixiviado bruto foi coletado no tanque de equalização da Estação Amarela de Tratamento de Efluentes da CTRS, que recebe lixiviado gerado na célula AC05 com idade de 9 anos.

\section{Operação do processo oxidativo avançado pelo reagente de Fenton}

Primeiramente $10 \mathrm{~L}$ de lixiviado bruto foram transferidos para um reator de mistura e teve seu $\mathrm{pH}$ ajustado para o $\mathrm{pH}$ de reação $(\mathrm{pH}=3,8)$ pela adição de ácido sulfúrico. Após ajuste do pH, foram adicionados sulfato ferroso hepta-hidratado (marca Synth) e peróxido de hidrogênio 30\% v/v (marca Vetec), empregando uma relação de DQO: $\mathrm{H}_{2} \mathrm{O}_{2}: \mathrm{FeSO}_{4} \cdot 7 \mathrm{H}_{2} \mathrm{O}=10: 6: 1$. Durante os 28 minutos de reação, o sistema foi mantido sob agitação mecânica com velocidade de rotação de 115 rpm. Alíquotas de amostras pré e pós-tratamento foram coletadas e caracterizadas . As condições operacionais foram otimizadas por Moravia (2010).

\section{Caracterização físico-química das amostras}

A caracterização das amostras de lixiviado bruto e pós-tratado contemplaram os seguintes parâmetros físico-químicos: cor real e aparente, demanda química de oxigênio (DQO), demanda bioquímica de oxigênio (DBO), carbono orgânico total (COT), potencial hidrogeniônico (pH), alcalinidade, série de sólidos (totais e suspensos), nitrogênio total e amoniacal, fósforo, cloretos e metais. As análises foram realizadas em conformidade com o Standard Methods for the Examination of Water and Wastewater (APHA et al., 2005).

O lodo gerado no POA/Fenton foi separado por decantação (com o auxílio da neutralização), homogeneizado e caracterizado quanto aos seguintes parâmetros físico-químicos: DQO, carbono total (CT), série de sólidos (totais) e ferro total.

\section{Avaliação da sedimentabilidade do lodo gerado do POA/Fenton}

O ensaio foi realizado por meio do teste de proveta (APHA et al., 2005). Adicionou-se a uma proveta de vidro com volume de $1 \mathrm{~L}$ uma quantidade com o mesmo volume de amostra (efluente pós-POA/Fenton), deixada em repouso até que a variação da altura da interface sólido/líquido fosse encerrada ou atingisse um tempo máximo de 2 horas, caracterizando o fim do processo de sedimentação. A cada 3 minutos a altura da interface sólido/líquido era obtida, determinando-se a curva de sedimentação. O valor da altura da interface utilizado para o cálculo do índice volumétrico do lodo (IVL) foi o correspondente ao tempo de 30 minutos. Determinou-se a concentração de sólidos suspensos totais da amostra para cálculo do IVL. Os valores de IVL foram determinados de acordo com a Equação 1.

$I V L=\frac{Z_{30^{\prime}} \times 10^{6}}{Z_{0}, x S S T}$ Equação 1

Onde:

IVL: índice volumétrico do lodo ( $\left.\mathrm{mL} . \mathrm{g}^{-1}\right)$;

$Z_{30}$ : altura da interface após 30 minutos do início do teste (m);

$Z_{0}:$ altura da interface no início do teste $(\mathrm{m})$;

SST: concentração de sólidos suspensos totais $\left(\mathrm{mg} \cdot \mathrm{L}^{-1}\right)$.

Por fim, determinou-se a velocidade de sedimentação como a média das velocidades atingida antes do tempo crítico de sedimentação. Os ensaios foram realizados com o efluente pós-POA/Fenton neutralizado em pH 7,0 a favor da melhor condição de sedimentabilidade.

\section{Unidade experimental}

A unidade experimental de POA/Fenton-MF é constituída de reator com capacidade para $10 \mathrm{~L}$, agitador de paletas, reservatórios de alimentação/coleta de permeado e concentrado (capacidade para $10 \mathrm{~L}$ cada), bomba elétrica de diafragma $\left(\mathrm{Q}_{\text {máx }}=456 \mathrm{~L} \cdot \mathrm{h}^{-1}\right)$ para bombeamento da alimentação, módulos de membrana polimérica tipo fibra oca de MF (marca: Pam Membranas) para a otimização (diâmetro de corte da membrana na faixa de 0,1 a 0,4 $\mu$ m e área efetiva de filtração igual a $\left.0,1 \mathrm{~m}^{2}\right)$ e para a operação $\left(1,0 \mathrm{~m}^{2}\right)$, manômetros, rotâmetros (alimentação e permeado). A unidade também apresenta circuito de limpeza por retrolavagem. A Figura 1 apresenta o diagrama esquemático da unidade experimental de POA/Fenton-MF.

\section{Otimização das condições operacionais do processo de MF}

\section{a) Avaliação do $\mathrm{pH}$ da alimentação}

O efluente pós-POA/Fenton foi submetido à MF em diferentes valores de $\mathrm{pH}(3,5$ e 7$)$, sendo avaliada a seletividade da membrana em relação à remoção dos principais constituintes do lodo: matéria orgânica, cor real, sólidos totais e ferro total. Foram verificadas também as condições de permeabilidade hidráulica da membrana com efluente em cada faixa de $\mathrm{pH}$ avaliada em comparação com os valores obtidos na operação com água microfiltrada

Para a avaliação da permeabilidade hidráulica, primeiramente foi realizada a compactação da membrana por meio da operação com água pura em sua alimentação e pressão constante de 1,5 bar até estabilização do fluxo. Em seguida, foi aplicada pressão decrescente na qual os valores obtidos para o fluxo de permeado $\left(\mathrm{L} \cdot \mathrm{h}^{-1} \cdot \mathrm{m}^{-2}\right)$ foram 


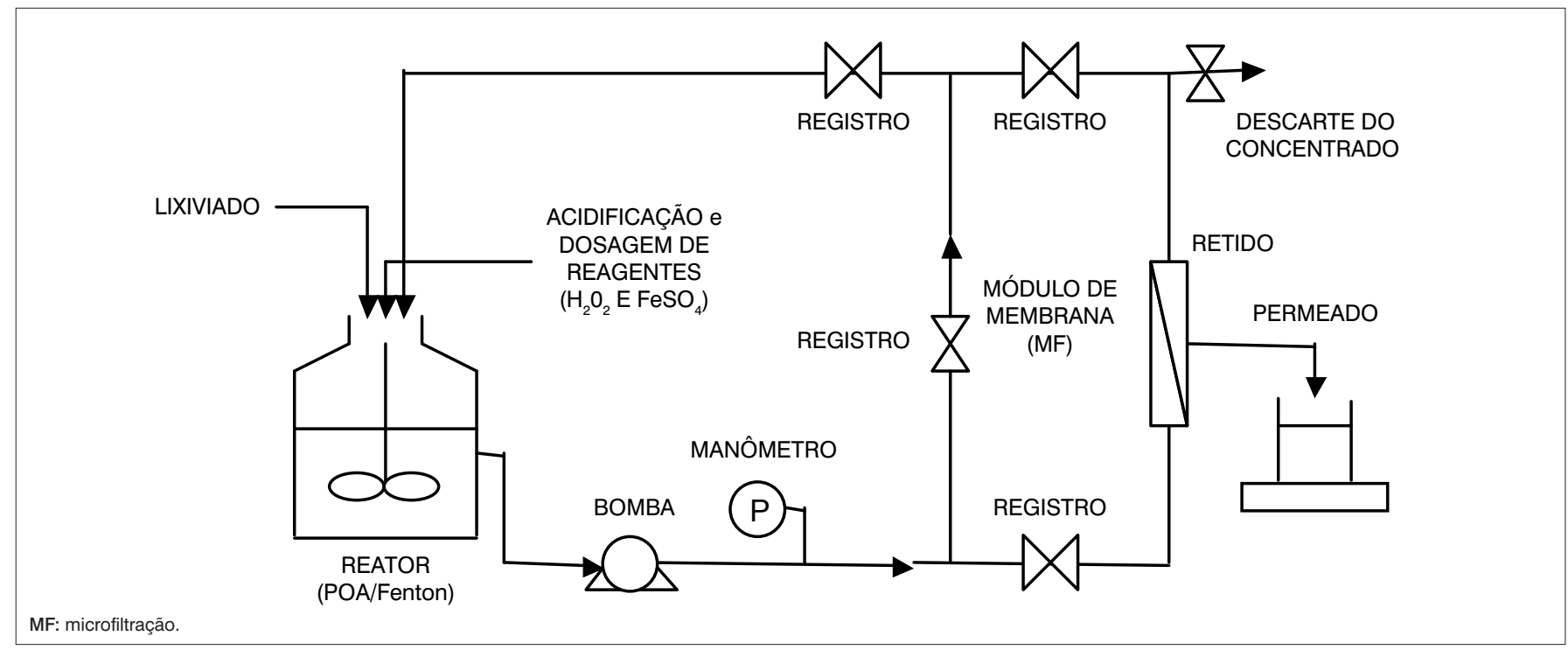

Figura 1 - Diagrama esquemático da unidade experimental de POA/Fenton- microfiltração.

representados em função da pressão aplicada. O coeficiente angular da reta ajustada aos pontos experimentais corresponde à permeabilidade hidráulica da membrana $\left(L \cdot h^{-1} \cdot \mathrm{m}^{-2} \cdot\right.$ bar $\left.^{-1}\right)$. Nos testes de permeabilidade, foi aplicado o menor número de Reynolds $\left(R_{e}\right)$ avaliado (2.600), objetivando a provável condição mais crítica de fluxo de permeado.

\section{b) Estabelecimento da pressão de operação}

O efeito da pressão de operação foi avaliado por meio da determinação da pressão crítica pelo método flux-step (BACCHIN et al., 2006). Para determinação da pressão crítica foram realizados ensaios nos quais a pressão foi fixada e o fluxo de permeado monitorado durante 18 minutos. A cada 3 minutos, a pressão era incrementada, sucessivamente, com respectivo acompanhamento do fluxo de permeado. Os valores de pressão avaliados foram de $0,25,0,5,0,75,1,0,1,25$ bar para o processo de MF.

\section{c) Estabelecimento do regime de escoamento}

O efeito do regime de escoamento foi avaliado variando-se a vazão de alimentação correspondente à variação dos regimes hidráulicos referentes aos $\mathrm{R}_{e}$ entre 2.600, 4.400 e 5.700 para o módulo de MF obedecendo às limitações da unidade experimental. Os ensaios foram realizados com o efluente em temperatura ambiente. Após o término de cada teste, os módulos foram submetidos aos processos de limpeza física e química até a recuperação total da membrana.

\section{Avaliação de técnicas de recuperação de fluxo na MF}

As técnicas de recuperação de fluxo empregadas foram a retrolavagem, o relaxamento e a recirculação - todas em condições estabelecidas. A vazão de retrolavagem aplicada foi equivalente a 1,5 vez o valor da vazão de alimentação. A duração da retrolavagem avaliada foi de 30 segundos em intervalos de 10 minutos para efeito comparativo. O relaxamento consistiu na despressurização do sistema e consecutiva cessão da vazão de recirculação. A duração da recirculação avaliada foi de 30 segundos em intervalos de 10 minutos para efeito comparativo. A recirculação foi por meio da despressurização do sistema e do aumento para o dobro da vazão de alimentação aplicada. A duração da recirculação avaliada foi de 30 segundos em intervalos de 10 minutos.

\section{Resultados}

\section{Caracterização físico-química do lixiviado bruto, sobrenadante pós-POA/Fenton e lodo gerado}

A Tabela 1 apresenta os valores dos parâmetros avaliados na caracterização físico-química do lixiviado bruto e pós-tratamento por POA/Fenton.

Em relação ao lixiviado bruto, observou-se uma alta concentração de matéria orgânica em termos de DQO ao longo de toda a caracterização. A baixa relação $\mathrm{DBO}_{5} / \mathrm{DQO}$ indica a baixa biodegradabilidade do lixiviado, confirmando um estado avançado de estabilização. O pH apresentou valor elevado em relação ao encontrado na literatura para lixiviados com características semelhantes (GOMES, 2009). Constatou-se também um baixo teor de sólidos em suspensão no lixiviado bruto. A concentração de metais pesados foi baixa não indicando indícios de atribuição de toxicidade por estes elementos. A concentração de ferro superior a de outros metais pode ser atribuída à intensa presença de óxidos de ferro no solo da região.

Em relação à eficácia do tratamento, para a remoção de cor e poluentes orgânicos, pode-se observar uma significativa remoção de poluentes após o tratamento. Observou-se uma eficiência de remoção de cor real e DQO de 76,4 e $76,7 \%$, respectivamente. A DQO persistente pode ser justificada pela formação de produtos intermediários estáveis que requerem um maior tempo para oxidação. Notou-se um aumento significativo da relação $\mathrm{DBO}_{5} /$ DQO. Esse fato se sustenta na intensa oxidação química dos compostos não biodegradáveis resultando na formação de produtos intermediários biodegradáveis justificando o incremento de DBO observado. Os baixos valores 
Tabela 1 - Caracterização físico-química do lixiviado bruto e efluente pós-POA/Fenton

\begin{tabular}{|c|c|c|c|c|c|c|c|c|c|}
\hline \multirow{2}{*}{ Parâmetros físico-químicos } & \multirow{2}{*}{ Unidade } & \multicolumn{4}{|c|}{ Lixiviado bruto } & \multicolumn{4}{|c|}{ Pós-POA/Fenton (sobrenadante) } \\
\hline & & Máx. & Mín. & Média & Desvio & Máx. & Mín. & Média & Desvio \\
\hline Cor real & $\mathrm{uH}$ & 568 & 420 & 467 & 60 & 128 & 92 & 110 & 12 \\
\hline Cor aparente & $\mathrm{uH}$ & 748 & 519 & 590 & 94 & 149 & 107 & 129 & 14 \\
\hline DQO & $m g \cdot L^{-1}$ & 2.428 & 2.220 & 2.354 & 94 & 588 & 471 & 548 & 41 \\
\hline $\mathrm{DBO}_{5}$ & $m g \cdot L^{-1}$ & 85 & 50 & 68 & 15 & 150 & 80 & 111 & 24 \\
\hline $\mathrm{DBO}_{5} / \mathrm{DQO}$ & - & 0,040 & 0,020 & 0,03 & 0,01 & 0,26 & 0,140 & 0,20 & 0,040 \\
\hline COT & $\mathrm{mg} \cdot \mathrm{L}^{-1}$ & 1.090 & 1.008 & 1.052 & 34 & 352 & 315 & 331 & 16 \\
\hline $\mathrm{pH}$ & - & 8,400 & 8,100 & 8,30 & 0,20 & 3,100 & 2,800 & 2,90 & 0,100 \\
\hline NTK & $\mathrm{mg} \cdot \mathrm{L}^{-1}$ & 1.172 & 1.036 & 1.105 & 57 & 1.137 & 1.032 & 1.085 & 45 \\
\hline $\mathrm{N}-\mathrm{NH}_{3}$ & $\mathrm{mg} \cdot \mathrm{L}^{-1}$ & 1.144 & 941 & 1.055 & 85 & 1.113 & 931 & 1.042 & 70 \\
\hline Cloretos & $\mathrm{mg} \cdot \mathrm{L}^{-1}$ & 2.333 & 2.033 & 2.190 & 124 & 2.266 & 1.966 & 2.127 & 114 \\
\hline Alcalinidade total & $m g \cdot L^{-1}$ & 6.041 & 4.791 & 5.263 & 557 & - & - & - & - \\
\hline Fósforo total & $\mathrm{mg} \cdot \mathrm{L}^{-1}$ & 19,600 & 11,300 & 14,50 & 3,70 & 11,200 & 2,700 & 6,40 & 3,100 \\
\hline ST & $\mathrm{mg} \cdot \mathrm{L}^{-1}$ & 9.408 & 8.120 & 8.801 & 591 & 11.318 & 8.554 & 9.743 & 1.002 \\
\hline STF & $\mathrm{mg} \cdot \mathrm{L}^{-1}$ & 6.002 & 5.114 & 5.595 & 370 & 4.384 & 3.144 & 3.715 & 445 \\
\hline STV & $m g \cdot L^{-1}$ & 3.736 & 2.452 & 3.227 & 614 & 7.207 & 5.332 & 6.044 & 688 \\
\hline SST & $\mathrm{mg} \cdot \mathrm{L}^{-1}$ & 26,500 & 17,000 & 22,40 & 4,10 & 4,700 & 3,000 & 3,90 & 0,700 \\
\hline SSF & $\mathrm{mg} \cdot \mathrm{L}^{-1}$ & 4,500 & 1,500 & 3,10 & 1,40 & 2,000 & 0,300 & 0,90 & 0,600 \\
\hline SSV & $\mathrm{mg} \cdot \mathrm{L}^{-1}$ & 22,000 & 15,500 & 19,30 & 2,80 & 4,000 & 2,000 & 3,00 & 0,800 \\
\hline Cádmio & $\mathrm{mg} \cdot \mathrm{L}^{-1}$ & 0,524 & $<0,005$ & 0,466 & 0,264 & 0,289 & $<0,005$ & 0,158 & 0,135 \\
\hline Chumbo & $\mathrm{mg} \cdot \mathrm{L}^{-1}$ & $<0,02$ & $<0,020$ & $<0,02$ & - & $<0,020$ & $<0,020$ & $<0,02$ & 0 \\
\hline Cromo & $\mathrm{mg} \cdot \mathrm{L}^{-1}$ & 0,490 & $<0,050$ & 0,23 & 0,23 & 0,320 & $<0,050$ & 0,16 & 0,130 \\
\hline Ferro & $\mathrm{mg} \cdot \mathrm{L}^{-1}$ & 10,350 & 2,200 & 5,68 & 3,60 & 318,900 & 178,380 & 269,91 & 53,020 \\
\hline Zinco & $\mathrm{mg} \cdot \mathrm{L}^{-1}$ & 0,140 & 0,070 & 0,10 & 0,03 & 0,330 & 0,020 & 0,14 & 0,110 \\
\hline
\end{tabular}

Máx: máximo; Mín: mínimo; DQO: demanda química de oxigênio; DBO: demanda bioquímica de oxigênio (Padrão); COT: carbono orgânico total; NTK: nitrogênio total Kjedhal; N-NH nitrogênio amoniacal; ST: sólidos totais; STF: sólidos totais fixos; STV: sólidos totais voláteis; SST: sólidos suspensos totais; SSF: sólidos suspensos fixos; SSV: sólidos suspensos voláteis

de pH são consequência da acidificação exigida pelo POA/Fenton, no qual o valor ótimo empregado para o lixiviado em estudo, conforme determinado por Moravia (2010), foi de 3,8. Ainda, as reações de $\mathrm{PO}_{4}^{-3}$ e $\mathrm{CO}_{3}^{-2} \mathrm{com}$ o radical hidroxila $\left({ }^{\circ} \mathrm{OH}\right)$ estão relacionadas com o decréscimo do $\mathrm{pH}$ em relação ao ajustado no início dos ensaios. O POA/Fenton não atuou satisfatoriamente na remoção de nitrogênio e fósforo, cujas concentrações permaneceram elevadas após o tratamento. Ainda, a concentração de sólidos totais no lixiviado após o processo oxidativo aumentou cerca de 11\%, indicando geração de lodo após a etapa de tratamento. A redução da concentração dos metais $\mathrm{Cr}$ e Cd após o POA/Fenton podem estar associada ao fenômeno de adsorção desses compostos no lodo gerado durante o tratamento.

Após decantação (sem o auxílio da neutralização) e posterior descarte da fração sobrenadante, o lodo foi separado, homogeneizado e caracterizado, sendo os resultados apresentados na Tabela 2.

A concentração de compostos de natureza orgânica no lodo pode ser confirmada pelos altos valores de DQO encontrados. Esse fato reforça ainda mais o acontecimento do fenômeno de adsorção durante o POA/ Fenton. Vale ressaltar ainda que esses valores foram corrigidos em função da interferência da concentração de ferro nos ensaios de DQO. Para correção da interferência da concentração de ferro foi determinada a correlação de concentração de ferro e DQO e, para dada concentração de ferro residual na amostra, era determinada a concentração de DQO equivalente ao ferro e esta era subtraída da fração de DQO da amostra. A concentração de CT no lodo foi elevada, situando-se próximo de 1.734 mg.L.-1, corroborando o afirmado anteriormente. Os valores de $\mathrm{pH}$ do lodo apresentaram-se compatíveis com os encontrados no sobrenadante, indicando assim
Tabela 2 - Caracterização físico-química do lodo gerado no POA/Fenton

\begin{tabular}{lccccc} 
Parâmetros & \multirow{2}{*}{ Unísico-químicos } & \multicolumn{4}{c}{ Lodo gerado no POA/Fenton } \\
\cline { 3 - 6 } & & Máx. & Mín. & Média & Desvio \\
DQO & $\mathrm{mg} \cdot \mathrm{L}^{-1}$ & 2.857 & 2.037 & 2.465 & 285 \\
CT & $\mathrm{mg} \cdot \mathrm{L}^{-1}$ & 2.070 & 1.335 & 1.734 & 304 \\
pH & - & 2,97 & 2,82 & 2,92 & 0,05 \\
ST & $\mathrm{g} . \mathrm{L}^{-1}$ & 13,50 & 12,30 & 12,90 & 0,40 \\
STF & $\mathrm{g} \cdot \mathrm{L}^{-1}$ & 6,80 & 5,70 & 6,40 & 0,40 \\
STV & $\mathrm{g} \cdot \mathrm{L}^{-1}$ & 7,00 & 6,20 & 6,50 & 0,30 \\
Ferro & $\mathrm{mg} \cdot \mathrm{L}^{-1}$ & 2.116 & 1.272 & 1.756 & 298
\end{tabular}

estabilidade da reação de oxidação. Em relação à natureza dos sólidos presentes no lodo, observou-se uma relação STV/ST igual a 0,50. A fração de STF pode ser atribuída à precipitação do ferro na forma de hidróxidos. A alta concentração de ferro no lodo reforça essa afirmativa (>1.200 mg. $\left.\mathrm{L}^{-1}\right)$.

\section{Avaliação da sedimentabilidade do lodo gerado do POA/Fenton}

O perfil de sedimentação do lodo gerado no POA/Fenton foi avaliado por meio das curvas de sedimentação e de velocidade de sedimentação obtidas no teste da proveta. Na Figura 2 são apresentadas as curvas respostas do teste.

Nota-se que o tempo crítico de sedimentação $(\Theta)$, ou seja, aquele a partir do qual se podem observar apenas duas fases no líquido, foi de 27 minutos. Foi observado também o término do tempo de compactação do lodo (tr) próximo aos 70 minutos de ensaio. A velocidade 

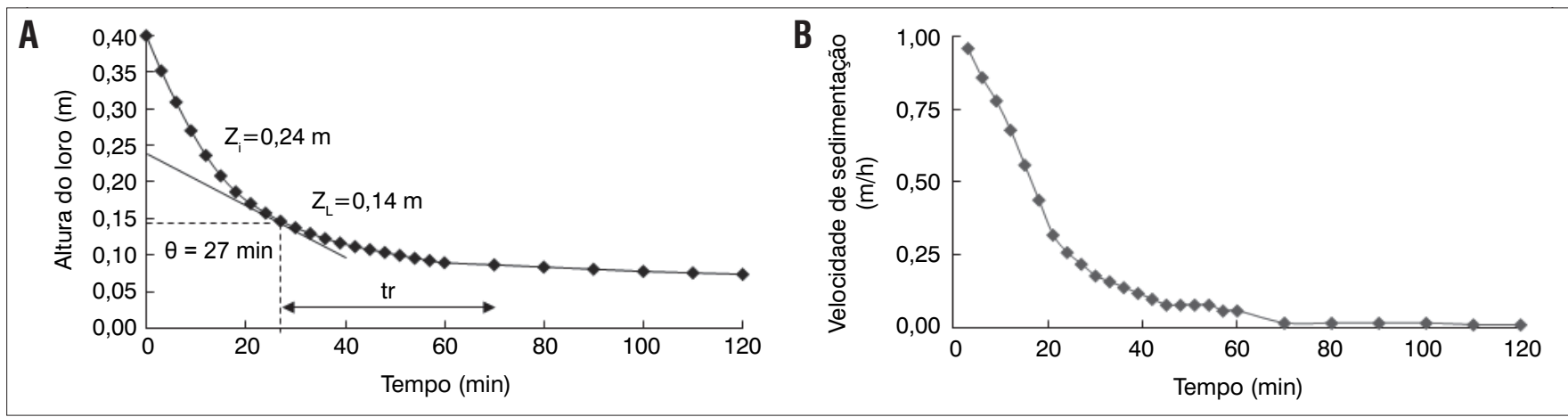

Figura 2 - Avaliação da sedimentabilidade do lodo gerado no POA/Fenton: (A) curva de sedimentação e (B) curva de velocidade de sedimentação.

média de sedimentação calculada com base na curva de velocidade de sedimentação foi de $0,56 \mathrm{~m} \cdot \mathrm{h}^{-1}$. Esses resultados indicam baixa sedimentabilidade do lodo gerado no POA/Fenton. De acordo com von Sperling (2001), o lodo pós-POA/Fenton apresentou classificação referente ao IVL como de péssima sedimentabilidade (> $\left.300 \mathrm{~mL} \cdot \mathrm{g}^{-1}\right)$. Na Tabela 3 é apresentado o resultado obtido.

\section{Otimização das condições operacionais do processo de MF}

\section{a) $\mathrm{pH}$ da alimentação}

Na Tabela 4 são apresentados os parâmetros de avaliação do processo de MF para diferentes valores de $\mathrm{pH}$ do efluente pós-POA/Fenton. Nota-se que a eficiência para remoção de cor real, ferro total, DQO e principalmente sólidos totais foi significativamente maior quando utilizado efluente neutralizado ( $\mathrm{pH}=7$ ). Esse resultado já era esperado, uma vez que em pH próximo ao neutro predominam espécies de ferro na forma de hidróxidos precipitáveis na forma de $\left[\mathrm{Fe}\left(\mathrm{H}_{2} \mathrm{O}\right)_{4}(\mathrm{OH})_{2}\right]$ que são retidos nos poros da membrana (NEYENS E BAEYENS, 2003). Observase ainda que, em pH ácido, o ferro encontra-se na forma solúvel, o que possibilita sua permeação por meio dos poros da membrana.

A Figura 3 mostra os resultados obtidos para a permeabilidade hidráulica da membrana para água e para o efluente pós-POA/Fenton em diferentes valores de $\mathrm{pH}$.

Como esperado, a permeabilidade do efluente com $\mathrm{pH}=7$ foi bastante reduzida apresentando valores próximos a $17 \mathrm{~L} \cdot \mathrm{m}^{-2} \cdot \mathrm{h}^{-1} \cdot \mathrm{bar}^{-1}$, enquanto que em $\mathrm{pH}=3$ atingiu valores superiores a $80 \mathrm{~L} \cdot \mathrm{m}^{-2} \cdot \mathrm{h}^{-1} \cdot \mathrm{bar}^{-1}$. A explicação para a redução da permeabilidade se deve às características reológicas do efluente que, em $\mathrm{pH}=7$, apresenta elevada concentração de sólidos totais principalmente devido a espécies de ferro precipitáveis e, consequentemente, obstrução dos poros da membrana. A formação de torta sobre a membrana pode atuar como uma membrana dinâmica que contribui para o aumento do grau de rejeição resultando numa maior remoção de compostos do efluente, o que pode ser evidenciado pela maior redução na DQO do permeado em $\mathrm{pH}=7$. O conjunto desses fatores sugere o emprego do efluente pós-POA/Fenton neutralizado como alimentação para a MF em função da maior seletividade da membrana.
Tabela 3 - Índice volumétrico do lodo gerado pós-POA/Fenton

\begin{tabular}{lcccc} 
Amostra & $\begin{array}{c}\mathrm{SST} \\
\left(\mathrm{mg} \cdot \mathrm{L}^{-1}\right)\end{array}$ & $\begin{array}{c}\mathrm{Z}_{0^{\prime}} \\
(\mathrm{m})\end{array}$ & $\begin{array}{c}\mathrm{Z}_{30^{\prime}} \\
(\mathrm{m})\end{array}$ & $\begin{array}{c}\mathrm{IVL} \\
\left(\mathrm{mL} \cdot \mathrm{g}^{-1}\right)\end{array}$ \\
\hline Pós-POA/Fenton + lodo & 1.067 & 0,400 & 0,137 & 321 \\
\hline
\end{tabular}

SST: concentração de sólidos suspensos totais; $Z_{0}$ altura da interface no início do teste; $\mathrm{Z}_{30}$ : altura da interface após 30 minutos do início do teste; IVL: índice volumétrico do lodo.

Tabela 4 - Caracterização do lixiviado bruto, alimentação e permeado do processo de microfiltração para diferentes valores de $\mathrm{pH}$

\begin{tabular}{|lcccc} 
& \multicolumn{4}{c}{ Microfiltração } \\
\cline { 2 - 5 } Amostra & $\begin{array}{c}\text { Cor real } \\
(\mathrm{uH})\end{array}$ & $\begin{array}{c}\text { Ferro } \\
\left(\mathrm{mg}^{-1}\right)\end{array}$ & $\begin{array}{c}\text { ST } \\
\left(\mathrm{mg} \cdot \mathrm{L}^{-1}\right)\end{array}$ & $\begin{array}{c}\text { DQO } \\
\left(\mathrm{mg}^{-1}\right)\end{array}$ \\
\hline Lixiviado bruto & 650 & 5,28 & 10.812 & 2.256 \\
\hline pH da alimentação=3 & & & & \\
\hline Alimentação (pós-POA/Fenton) & $3.249,00$ & 585,17 & 12.586 & 920 \\
\hline Permeado & 182,40 & 79,48 & 3.786 & 861 \\
\hline Eficiência (\%) & 94,39 & 86,42 & 69,92 & 6,35 \\
\hline pH da alimentação=5 & & & & \\
\hline Alimentação (pós-POA/Fenton) & $3.584,00$ & 600,77 & 12.538 & 1.037 \\
\hline Permeado & 149,10 & 18,64 & 3.381 & 800 \\
\hline Eficiência (\%) & 95,84 & 96,90 & 73,03 & 22,87 \\
\hline pH da alimentação=7 & & & & \\
\hline Alimentação (pós-POA/Fenton) & $3.238,00$ & 578,51 & 12.326 & 1.029 \\
\hline Permeado & 127,90 & 1,12 & 1.193 & 770 \\
\hline Eficiência (\%) & 96,05 & 99,81 & 90,32 & 25,18 \\
\hline
\end{tabular}

ST: sólidos totais; DQO: demanda química de oxigênio.

\section{b) Estabelecimento da pressão de operação}

Adotando pH da alimentação neutro, a pressão de operação foi avaliada por meio da avaliação da pressão crítica. A pressão crítica é definida como aquela em que a relação entre o aumento da pressão e o aumento do fluxo de permeado se torna não linear, ou seja, a partir da qual se observa a incrustação da membrana. Na Figura 4 é apresentada a relação entre fluxo de permeado e pressão de operação usada para determinação de pressão crítica do processo de MF. A vazão de alimentação $\left(Q_{a}\right)$ empregada foi de 2,4 L.min'-1 $\left(R_{e}=2.600\right)$.

Observou-se que o fluxo decai imediatamente após o início da permeação, indicando assim incrustação precoce da membrana. Essa incrustação provavelmente se deve às características da alimentação e representa as condições reais do processo nessa aplicação. Provavelmente, em função dos sólidos coloidais, as forças convectivas na direção da superfície da membrana acarretadas pelo gradiente de pressão, mesmo que baixo, já são 
suficientes para elevar a concentração de sólidos próximos a camada seletiva da membrana e, com isso, favorecer a formação de torta e a precipitação química de compostos solúveis. Dessa forma, para uma operação sustentável do sistema, sugere-se a operação com pressão de 0,25 bar para a MF.

\section{c) Estabelecimento da regime de escoamento}

A verificação dos efeitos do regime de escoamento sobre a permeabilidade operacional no sistema de MF foi realizada empregando

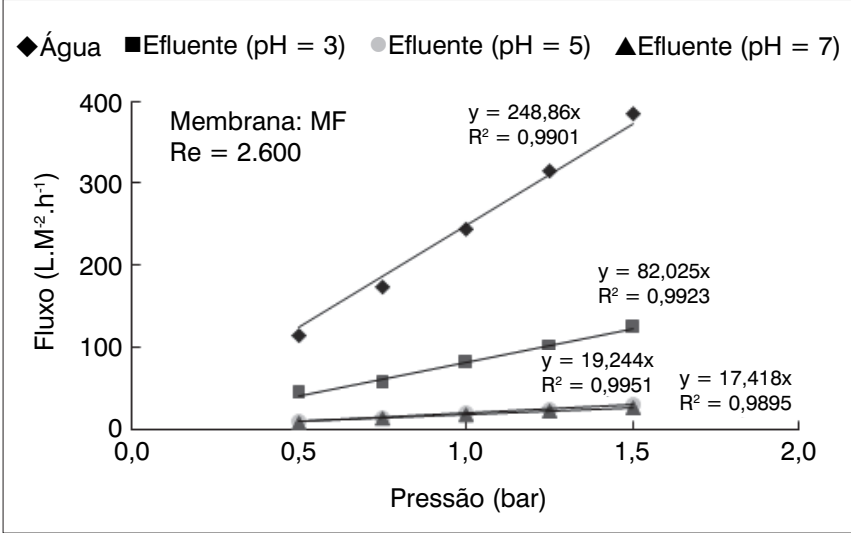

Figura 3 - Permeabilidade hidráulica da membrana para água e efluente pós-POA em diferentes valores de $\mathrm{pH}$.

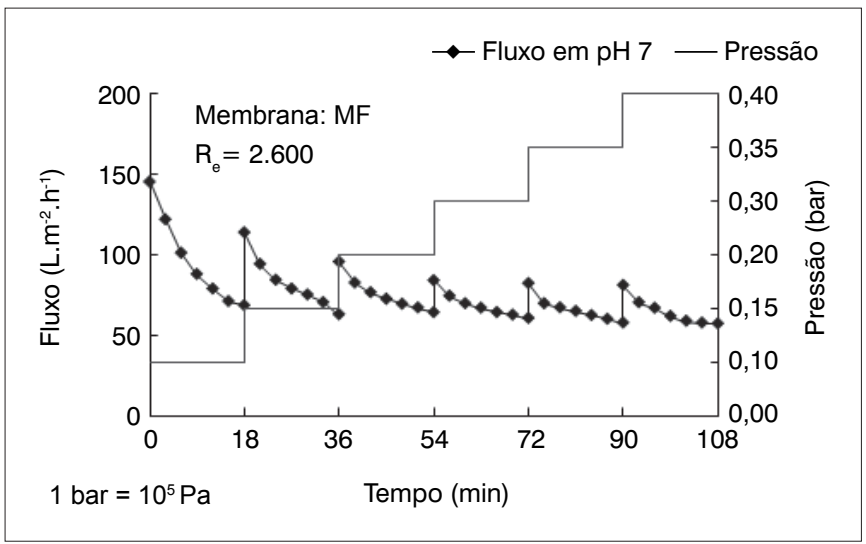

Figura 4 - Relação entre fluxo de permeado e pressão de operação para determinação de pressão crítica no processo de microfiltração. diferentes vazões de alimentação, respectivas aos regimes hidráulicos relativos aos $R_{e}$ de $2.600,4.400$ e 5.700. A Figura 5 mostra a permeabilidade operacional da MF quando operadas em diferentes $\mathrm{R}_{e}$. Esses ensaios foram realizados para pressões otimizadas ( 0,25 bar).

De acordo com os resultados, o fluxo estabilizado de permeado da MF aumentou quando operado com regime de escoamento com $R_{e}$ de 5.700 em relação ao $R_{e}$ de 2.600. A permeabilidade operacional foi maior para $\mathrm{R}_{\mathrm{e}}$ mais alto. Esses resultados podem ser explicados pela maior turbulência e maior cisalhamento próximo à superfície da membrana, o que acarreta no maior arraste e, consequentemente, menor concentração de partículas próximas à camada seletiva, elevando-se assim o fluxo de permeado. Os resultados também mostraram um coeficiente de recuperação de permeado próximos para os regimes de escoamento avaliados. O regime de escoamento ótimo sugerido do sistema de MF foi equivalente a $\mathrm{R}_{\mathrm{e}}$ igual a $5.700\left(Q_{a}=2,4 \mathrm{~L} \cdot \mathrm{min}^{-1}\right)$.

\section{Avaliação de técnicas de recuperação de fluxo na MF}

Na Figura 6 são apresentados os perfis do fluxo de permeado em relação ao tempo de operação empregando as diferentes técnicas de recuperação de fluxo aplicadas durante 30 segundos em intervalos

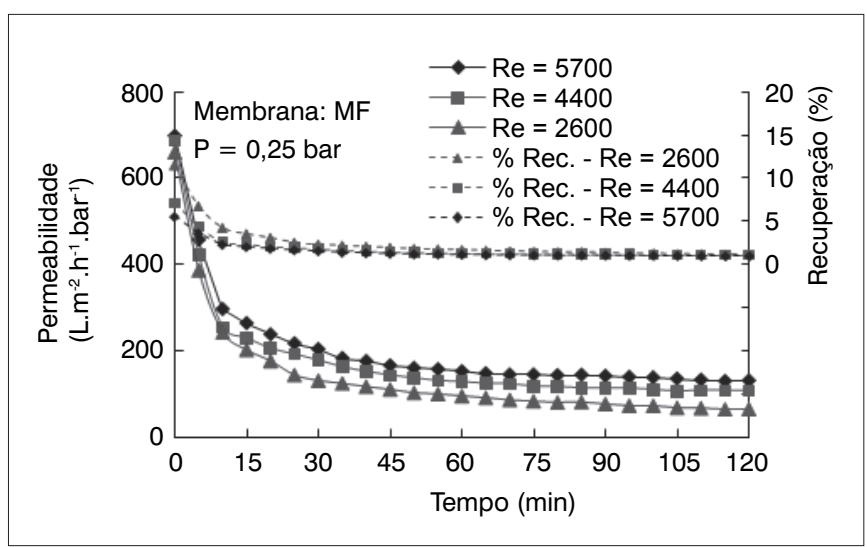

Figura 5 - Permeabilidade operacional para diferentes regimes de escoamento durante a operação da microfiltração.
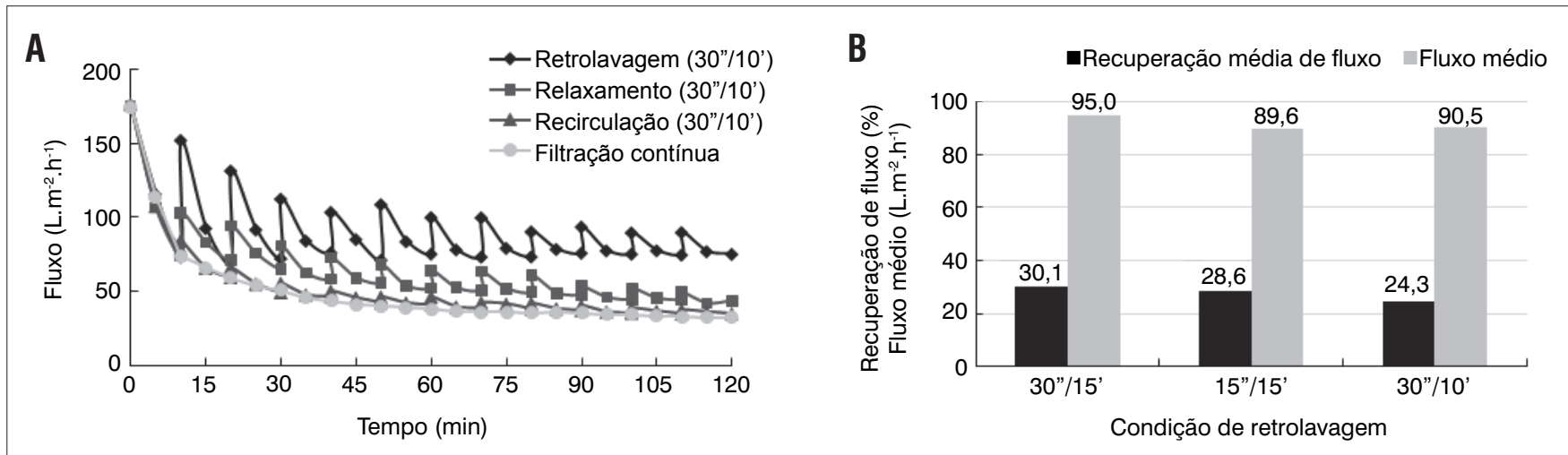

Figura 6 - (A) Perfil de fluxo permeado em diferentes modos de operação; (B) eficiência de recuperação de fluxo de permeado e fluxo médio das diferentes condições de retrolavagem. 
de 10 minutos e a eficiência de recuperação de fluxo de permeado e fluxo médio das diferentes condições de retrolavagem.

Observa-se que todas as técnicas avaliadas permitem a recuperação do fluxo de permeado, sendo a retrolavagem a mais eficiente, pois apresentou recuperação média de fluxo superior a 18\%. O uso da recirculação no mesmo sentido da alimentação foi pouco efetivo. Uma possível justificativa é o estado precipitado das partículas na superfície da membrana, consistindo numa torta adensada predominada por forças de interações físicas e químicas. Já a técnica de relaxamento apresentou recuperação média de fluxo pouco superior à recirculação. Os resultados também mostraram baixa influência da frequência e duração da retrolavagem na recuperação de fluxo de permeado. Sendo assim, o emprego da retrolavagem em intervalos de 15 minutos com duração de 15 segundos apresentou-se como condição mais atrativa em função da menor demanda de permeado.

\section{Conclusão}

Os resultados apontaram que o lixiviado em estudo possui características refratárias, reforçando as vantagens do tratamento baseado em processos físico-químicos. Observou-se que o POA/Fenton, em suas condições otimizadas, apresentou elevada eficiência de remoção de DQO $(76,7 \%)$ e cor real (76,4\%). Foram detectados compostos de natureza orgânica no lodo gerado ( $\mathrm{DQO}=2.465 \mathrm{mg} \cdot \mathrm{L}^{-1}$ ) provavelmente oriundos da adsorção durante a reação e elevada concentração de ferro $\left(1.757 \mathrm{mg} \cdot \mathrm{L}^{-1}\right)$. O lodo pós-POA/Fenton apresentou valores do IVL superior a $300 \mathrm{~mL} . \mathrm{g}^{-1}$, o que torna sua separação por sedimentação pouco atrativa. Os resultados mostraram que a otimização do processo de MF contribuiu significativamente para o aumento da seletividade da membrana e produtividade de efluente tratado. A seletividade da membrana foi maior para $\mathrm{pH}$ neutro da alimentação $(7,0)$. Em relação à pressão crítica, esta não foi observada provavelmente em função das características da alimentação, a qual, mesmo com pressão de operação muito baixa $(0,25$ bar), já foi suficiente para condicionar a formação de torta. Constatou-se também que o aumento do $\mathrm{R}_{\mathrm{e}}$ de 2.600 para 5.700 proporcionou um incremento no fluxo de permeado, sendo que este aumentou de 16,3 para 32,7 L/h (200\%) quando operado com pressão de 0,25 bar. Em relação à recuperação de fluxo da membrana, a técnica mais eficaz foi a retrolavagem aplicada em intervalos de 15 minutos com duração de 15 segundos.

\section{Referências}

APHA; AWWA; WEF. Standard methods for the examination of water and wastewater. 21 ed. Washington, DC: American Public Health Association, 2005.

BACCHIN, P.; AIMAR, P.; FIELD, R.W. Critical and sustainable fluxes: theory, experiments and applications. Journal of Membrane Science, $v$. 15, p. 42-69, 2006.

BAKER, R.W. Membrane technology and applications. 2 ed. England: John Wiley \& Sons, 2004.

BULL R.A.; ZEFF, J.D. Hydrogen peroxide in advanced oxidation process for treatment of industrial process and contaminated groundwater. IN: ECKENFELDER, W.W.; BOWERS, A.R.; ROTH, J.A. Chemical oxidation: technologies for the nineties. Lancaster: Technomic, 1991. p. 26-36.

CHAMARRO, E.; MARCO, A.; ESPULGAS, S. Use of Fenton reagent to improve organic chemical biodegradability. Water Research, v. 35, n. 4, p. 1047-1051, 2001.

CHIU, T.Y.; JAMES A.E. Sustainable flux enhancement in non-circular ceramic membranes on wastewater using the Fenton process. Journal of Membrane Science, n. 279, p. 347-353, 2006.

ENGLEHARDT, J. D. et al. Options for Managing Municipal Landfill Leachate: Year 1 Development of Iron-Mediated Treatment Processes. Tech. Rep., Florida Center for Solid and Hazardous Waste Management, EUA, 2006

GOMES L.P. et al. Estudos de caracterização e tratabilidade de lixiviados de aterros sanitários para as condições brasileiras. IN: GOMES L.P.
(ORG.) Resíduos sólidos urbanos: aterro sustentável para municípios de pequeno porte. Rio de Janeiro: Rima ABES, 2009. p. 26-59.

HABERT, A.C.; BORGES, C.P.; NOBREGA, R. Escola Piloto em Engenharia Química: processos de separação com membranas. Rio de Janeiro: COPPE/UFRJ, 2006.

KURNIAWAN, T.A.; LO, W.; CHAN, G.Y.S. Physico-chemical treatments for removal of recalcitrant contaminants from landfill leachate. Journal of Hazardous Materials, v. 129, p. 0-100, 2006

LI, W., ZHOU, Q.; HUA, T. Removal of organic matter from Landfil Leachate by advanced oxidation processes: a review. International Journal of Chemical Engineering, 2010, doi:10.1155/2010/270532.

MORAVIA, W.G. Avaliação do tratamento de lixiviado de aterro sanitário através de processo oxidativo avançado conjugado com sistema de separação por membranas. 261p. Tese (Doutorado em Engenharia Sanitária e Ambiental) - Universidade Federal de Minas Gerais, Belo Horizonte, 2010

NEYENS, E.; BAEYENS. J. A review of classic Fenton's peroxidation as an advanced oxidation technique. Journal of Harzadous Materials, v. 28, p. 33-50, 2003.

ORDÓNEZ, J.A. Tecnología de alimentos: componentes dos alimentos e processos. Porto Alegre: Artmed, 2005.

VON SPERLING, M. Dimensionamento de lodos ativados por batelada utilizando os princípios da teoria do fluxo de sólidos. Engenharia Sanitária e Ambiental, v. 6, 2001. 